Objectives The objective of the quality improvement project was to increase the utilization of TWIST score, a validated clinical scoring system from $0 \%$ to $80 \%$ over 12 months period for children evaluated for testicular torsion, as a means of increasing the number of patients with CDOR60.

Methods Deploying the Institute for Healthcare Improvement Model for Improvement, we formulated an aim statement and identified key drivers. Of our interventions, successful implementation of the TWIST score in the emergency department (ED), with a guideline to support earlier notification of urology for high risk patients with testicular pain, was determined to be the highest impact intervention. We educated providers and gave biweekly feedback about guideline adherence, created order panels and documentation tabs in our electronic medical record to encourage documentation.

Results Between September 2018 and April 2019, TWIST score documentation improved to $>80 \%$ and was sustained at that rate for 4 months. After implementation of the guideline, the critical diagnosis to OR time of 60 minutes or less was achieved in 12 out of 16 patients diagnosed with testicular torsion in the ED [75\%] compared to our baseline of $33 \%$.

Conclusions A quality improvement project to improve the timeliness of care for children with testicular torsion resulted in delivery of expedited surgical care for these patients.

\section{PLANNED COMMUNITY PARAMEDICINE VISIT PROGRAM REDUCE ED VISITS AND READMISSIONS}

${ }^{1}$ Amanda Ascher, ${ }^{2}$ Hanan Cohen, ${ }^{3}$ Zoë Stopak-Behr, ${ }^{4}$ Irene Kaufmann. ${ }^{1}$ Bronx Partners for Healthy Communities, SBH Health, USA; ${ }^{2}$ Empress, USA; ${ }^{3}$ SBH Health System, USA; ${ }^{4} B r o n x$ Partners for Healthy Communities, SBH Health, USA
Background Many patients with multiple chronic conditions have challenges in understanding, accessing and navigating the healthcare system, leading to frequent ED use, admissions and readmissions. We piloted a planned Community Paramedicine Visit Program (CPVP) to patients at risk for repeated ED visits or admissions, in an effort to reduce hospital utilization in a community safety-net hospital.

Objectives Reduce avoidable hospital use by identifying highrisk patients with patterns of high utilization and referring them to CPVP.

Methods This pilot was conducted in The Bronx, NY with a single EMS agency and a single safety-net hospital, focused on Medicaid patients as part of a NYS Medicaid waiver. Initial challenges of identifying and referring high-risk patients were addressed using PDSA ramps. Analysis looked at hospital use for each of the 43 unique patients referred into the CPVP and compared equal time periods pre- and post-referral for each patient. The analysis does not include patients who refused referral or accepted referral but refused enrollment into CPVP.

Results The cohort had 160 visits to the hospital (inclusive of emergency department visits, admissions and readmissions) in the 1 to 6 months prior to their referral. The same cohort of patients had only 65 hospital visits in the 1 to 6 months after their engagement in the CPVP; this represents a $59.4 \%$ reduction in hospital use and a $59.4 \%$ decrease in the average number of visits per patient.

Conclusions The Community Paramedicine Visit Program decreased hospital use by high-risk patients. Team-based QI efforts help engage more patients in this valuable program.

10.1136/bmjoq-2019-ihi.3

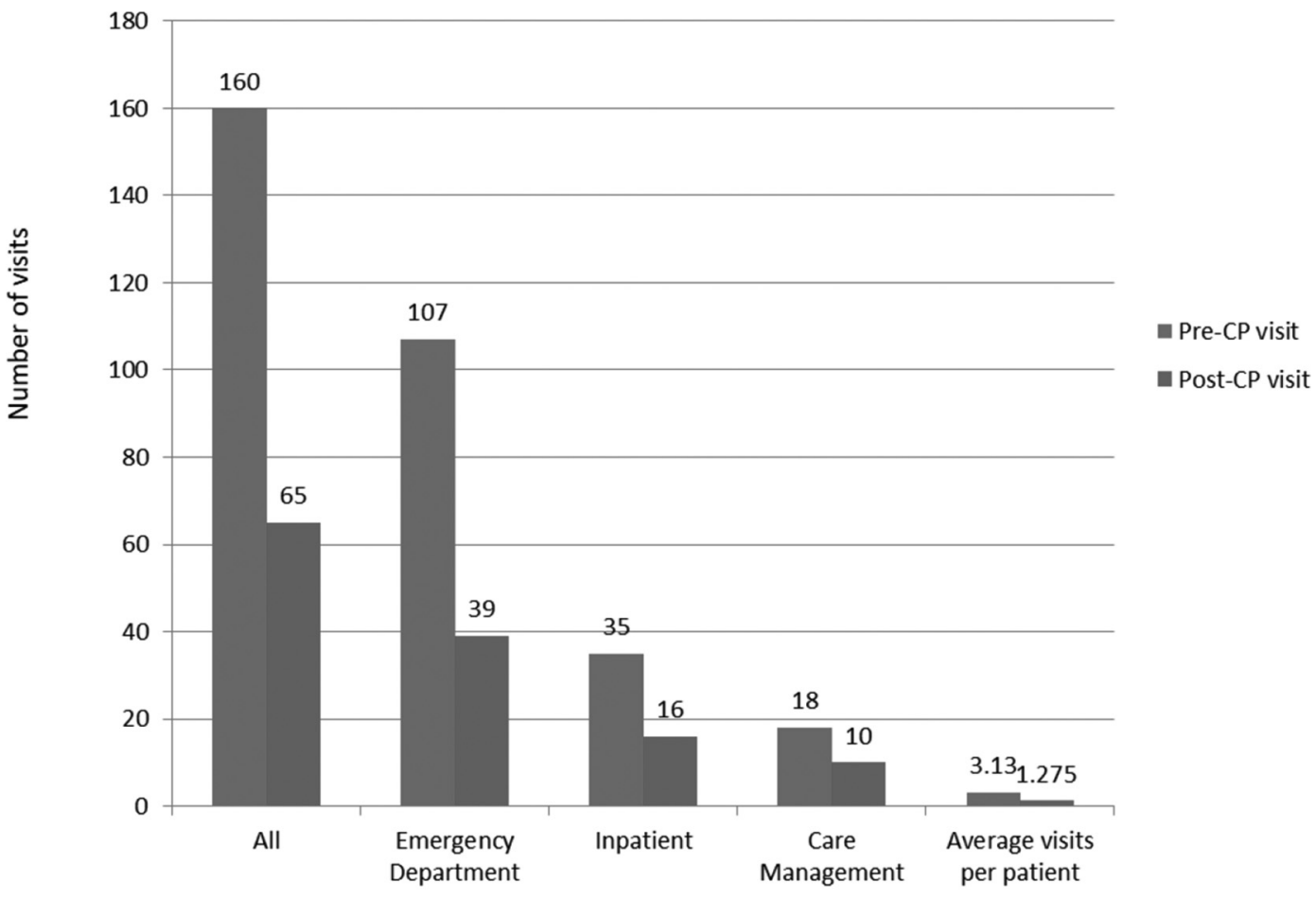

Referral Source 